\title{
Estudo preliminar de adaptação e validação do Inventário de Psicoterapia Positiva numa Amostra da População Portuguesa
}

\section{Preliminary study of adaptation and validation of the Inventory of Positive Psychotherapy for a Portuguese population's sample}

\section{Marlene Ferreira (1)}

Ana Pereira (1)

Ana Prior (1)

Carla Fonte (1)

(1) Universidade Fernando Pessoa, Portugal

Recebido: 05/08/2020; Revisto: 26/09/2020; Aceite: 27/10/2020.

https://doi.org/10.31211/rpics.2020.6.2.185

\section{Resumo}

Contexto e Objetivo: A psicoterapia positiva alicerça-se concetualmente no estudo científico de emoções positivas, traços individuais positivos e pontos fortes. O objetivo é ajudar as pessoas a aprenderem que podem crescer como resultado de suas experiências, mesmo que a experiência seja traumática. Trata-se de um método psicoterapêutico que se foca na construção de emoções positivas, forças e significado na vida dos indivíduos para diminuir e prevenir a psicopatologia e promover a felicidade. Como ferramenta de avaliação, o Inventário de Psicoterapia Positiva (IPP) oferece aos profissionais de saúde mental a oportunidade de ver os resultados do processo terapêutico. Assim, o objetivo do presente estudo é conhecer as qualidades psicométricas do IPP numa amostra da população portuguesa. Métodos: Trata-se de um estudo metodológico, de carácter exploratório e descritivo, que visa a tradução-retroversão e análise das propriedades psicométricas (fidelidade e validade) recorrendo à administração do IPP, da Escala de Ansiedade, Depressão e Stress de 21 itens e do Mental Health Continuum - Short Form. A amostra foi composta por 247 participantes entre os 18 e 69 anos. Resultados: A versão traduzida e adaptada da escala cumpriu os critérios de equivalência semântica e apresentou um nível elevado de consistência interna ( $\alpha$ de Cronbach $=0,97$ ), altos valores de correlação entre formas $(r=0,92)$, assim como uma correlação elevada do item com o total do teste. 0 inventário ficou composto por 25 itens e explicado por três fatores, cumprindo os critérios de validade convergente e divergente. Conclusões: $\mathrm{O}$ estudo preliminar do IPP apresentou boas qualidades psicométricas. Sugere-se o alargamento da amostra para sustentação dos resultados obtidos.

Palavras-Chave: Adaptação; Inventário de Psicoterapia Positiva; Psicoterapia positiva; Validação.

\section{DI\&D | ISMT}

rpics@ismt.pt https://rpics.ismt.pt
Publicação em Acesso Aberto

(c)2020. O(s) Autor(es). Este é um artigo de acesso aberto distribuído sob a Licença Creative Commons Attribution, que permite uso, distribuição e reprodução sem restrições em qualquer meio, desde que o trabalho original seja devidamente citado.
Marlene Ferreira

Rua Gentios, porta $142,1^{\circ}$ Esq.

4580-732, Sobrosa - Paredes, Portugal

E-mail: marlene.silva.ferreira11@gmail.com 


\begin{abstract}
Background and Aim: Positive psychotherapy is conceptually based on the scientific study of positive emotions, positive individual traits, and strengths. The goal is to help people learn that they can grow due to their experiences, even if the experience is traumatic. It is a psychotherapeutic method that focuses on building positive emotions, strengths, and meaning in individuals' lives to decrease and prevent psychopathology and promote happiness. As an assessment tool, the Positive Psychotherapy Inventory (PPTI) offers mental health professionals the opportunity to see the therapeutic process's results. The present study's objective is to know the psychometric qualities of the PPTI in a Portuguese sample. Method: This is a methodological study of an exploratory and descriptive character, which aims at translation-retroversion and analysis of psychometric properties (fidelity and validity) using the administration of the PPTI, the Depression Anxiety Stress Scales - 21 items, and Mental Health Continuum - Short Form. The sample consisted of 247 participants between 18 and 69 years old. Results: The translated and adapted version of the scale met the criteria of semantic equivalence and had a high level of internal consistency (Cronbach's $\alpha=0.97$ ), high values of correlation between forms $(r=0.92)$, as well as a high correlation of the item with the test total. The inventory consisted of 25 items and was explained by three factors, meeting the convergent and divergent validity criteria. Conclusions: The preliminary PPTI study showed good psychometric qualities. It is suggested enlarging the sample to support the results obtained.
\end{abstract}

Keywords: Adaptation; Positive psychotherapy; Positive Psychotherapy Inventory; Validation.

\title{
Introdução
}

Durante muitos anos, a Psicologia, e especificamente a Psicoterapia, foi encarada como o local onde os utentes vão falar sobre os seus problemas e onde o foco foi quase sempre na reparação de dimensões negativas como sintomas, traumas, feridas, défices e distúrbios (Seligman et al., 2006). Esta ênfase dada aos problemas permitiu que a psicologia ajudasse as pessoas a melhorar de uma série de psicopatologias e aliviasse o seu sofrimento. No entanto, não sendo a saúde mental a mera ausência de sintomas começou entre a comunidade científica a emergir o interesse pelo estudo e compreensão das dimensões psicológicas mais positivas e funcionantes. Embora noções como individualização e autorrealização, pleno funcionamento, maturidade e saúde mental positiva sejam pontuais na literatura, estas foram vistas como subprodutos do alívio dos sintomas ou como luxos clínicos que, nesta era apressada não há tempo para abordar (Jahoda, 1958; Allport, 1961; Rogers, 1961; Maslow, 1971 e Baumeister et al., 2001, citados por Rashid \& Seligman, 2019).

Com o intuito de proporcionar uma mudança deste foco, emerge o movimento da Psicologia Positiva que procura analisar e compreender os construtos psicológicos mais positivos, tais como o bem-estar, a esperança, o otimismo, a felicidade e a resiliência. Assim, contrastando com a Psicologia clássica, este ramo visa a promoção do funcionamento ótimo da pessoa, enfatizando a compreensão dos fatores que se constituem como pontes fortes, promovendo a sua saúde mental, bem-estar subjetivo e felicidade (Kun et al., 2017). Deste modo, segundo Rashid (2015), a Psicologia Positiva sobrepõe as forças do indivíduo às suas fraquezas, de modo a enfatizar as melhores coisas da vida, habilidades e pontos fortes do indivíduo e tirar partido dos momentos de bem-estar em prol da patologia que possa estar associada.

Tendo em conta a anterior definição, Seligman propõe que, inerente ao conceito de felicidade plena existem três dimensões: vida significativa, vida agradável e vida comprometida (Sirgy \& Wu, 2009). De modo concreto, os autores defendem que a vida significativa valoriza o envolvimento com o outro e com atividades onde a atenção está completamente focada no seu desenvolvimento, tendo assim inerente o conceito de flow. 0 sujeito é capaz de investir e potenciar as suas forças nas relações e atividades em que está envolvido. De acordo com os mesmos autores, a dimensão da vida agradável tem inerente emoções positivas acerca do presente, do 
passado e do futuro, valorizando as experiências precoces como fonte de desenvolvimento, ainda que possam ser menos positivas. Assim, o indivíduo é capaz de fazer perdurar a intensidade e duração das emoções positivas subjacentes às suas experiências de vida diária. Por último, no que diz respeito à vida comprometida, são valorizadas as capacidades do indivíduo em usar as suas forças e talentos ao serviço de algo ou alguém, de forma a alcançar o reconhecimento através do compromisso e satisfação pela entrega à comunidade ou ao outro (Seligman et al., 2006; Sirgy \& Wu, 2009).

Ainda baseado na psicologia positiva, Seligman (2012, tal como citado por Kun et al., 2017) desenvolveu o modelo PERMA (Positive Emotion; Engagement; Relationship; Meaning; Accomplishment), cujas bases teóricas assentam nas três dimensões descritas anteriormente. Segundo o autor, o bem-estar é composto por cinco elementos: emoções positivas, compromisso, relacionamentos, significado/propósito e realização. Sinteticamente, as emoções positivas conjugam a premissa de que bons sentimentos motivam as ações humanas e que melhoram o desempenho das diversas atividades que o indivíduo realiza ao longo do seu dia-adia, fortalecendo relacionamentos, criando otimismo e esperança no futuro (Kun et al., 2017). O compromisso refere-se ao apego, ao envolvimento e à concentração inerente às atividades sendo elas de lazer ou de trabalho. Descreve um estado de imersão completa no presente, tendo assim subjacente o conceito de flow (Asebedo \& Seay, 2014; Kun et al., 2017). Inerente aos relacionamentos encontra-se a busca de conexões saudáveis, positivas e gratificantes com os outros, com o intuito de manter um contacto físico e emocional. 0 estabelecimento destas relações conduz a um estreitamento de laços com familiares, amigos ou colegas, melhorando o próprio bem-estar, nutrindo o sentimento de pertença do indivíduo (Kun et al., 2017). o elemento do significado e propósito envolve o uso das forças do indivíduo, não só em benefício de si mesmo, mas na sua orientação para o cumprimento de metas que são consideradas importantes. Parte da premissa de que se está no seu melhor quando se se dedica a algo maior que si mesmo. Deste modo, o significado e propósito envolve atividades voluntárias, pertença a comunidades ou grupos cívicos com um propósito que orienta os indivíduos para algo maior (Kun et al., 2017; Rashid \& Seligman, 2018). O quinto elemento do modelo PERMA é a realização e subentende a busca pelo sucesso, domínio e conquista de objetivos. Deste modo, o sentido de realização orienta uma vida produtiva e significativa, trilhado pelo sujeito com o intuito de alcançar o bem-estar. Assim as conquistas ao longo da vida devem ser celebradas com um sentimento de realização, uma vez que o sucesso foi alcançado pelo próprio indivíduo (Asebedo \& Seay, 2014; Kun et al., 2017; Rashid \& Seligman, 2018). Alicerçado nestes princípios conceptuais tem emergido recentemente, uma abordagem diferente para o tratamento dos problemas psicológicos bem como para a intervenção com indivíduos sem qualquer problema ao nível da saúde mental. Esta designa-se de psicoterapia positiva (PPT) e concentra-se nas habilidades internas e pontos fortes dos indivíduos para lidar com problemas que estes enfrentam na sua vida, em vez de focar apenas nos sintomas e dificuldades. A PPT ajuda as pessoas a identificar o que está a acontecer de funcionante na sua vida. Variadas evidências empíricas têm vindo a confirmar que a PPT ajuda as pessoas a experimentar felicidade na sua vida que pode melhorar o bem-estar e saúde mental de uma pessoa. Deste modo, mediante a exposição dos conteúdos teóricos anteriormente apresentados, depreende-se que as três dimensões teóricas subjacentes ao construto da felicidade plena agrupam os cinco elementos do modelo PERMA, proposto por Seligman (Guney, 2011). É neste contexto conceptual, empírico e prático que emerge o Inventário de Psicoterapia Positiva (IPP) que é composto por vários itens que constituem o modelo PERMA, mas que se agrupam nas três grandes dimensões teóricas - vida agradável, vida comprometida e vida significativa. Este inventário é a medida principal para avaliar o bem-estar do utente, com base no modelo do bem-estar 
PERMA. O IPP avalia o bem-estar em termos de emoções positivas, envolvimento, relacionamentos, significado e realização (Rashid \& Seligman, 2018). Este inventário tem agora recentemente despertado o interesse na comunidade científica, contando já com a sua adaptação para turco (Guney, 2011), persa (Khanjani et al., 2014) e alemão (Wammerl et al., 2015).

O objetivo do presente estudo foi traduzir e adaptar o IPP para a população portuguesa e verificar as suas propriedades psicométricas, testadas ao nível da confiabilidade e validade. Trata-se de um estudo preliminar de apresentação das qualidades psicométricas obtidas.

\section{Método}

\section{Participantes}

Os participantes da presente investigação foram selecionados através de uma amostragem intencional, não probabilística, nomeadamente, por conveniência (Ribeiro, 2010).

A recolha de dados respeitou os critérios de inclusão e exclusão previamente definidos nomeadamente (a) ter 18 anos. Deste modo, tendo em consideração a Tabela 1, a amostra total foi constituída por 247 participantes (180 do sexo feminino e 67 do sexo masculino), com idades compreendidas entre os 18 e os 69 anos $(M=36,89 ; D P=$ $11,4)$, detentores, na maioria, de mestrado $(35,6 \%)$. Relativamente ao estado civil, registou-se um total de $38,5 \%$ solteiros e a mesma percentagem de casados.

\section{Tabela 1}

Características Sociodemográficas da Amostra ( $\mathrm{N}=247)$

\begin{tabular}{|c|c|c|c|}
\hline & & $n$ & $\%$ \\
\hline \multirow{2}{*}{ Sexo } & Feminino & 180 & 72,9 \\
\hline & Masculino & 67 & 27,1 \\
\hline \multirow{7}{*}{ Habilitações literárias } & 10 Ciclo & 0 & 0 \\
\hline & 2을 Ciclo & 6 & 2,4 \\
\hline & 3으 Ciclo & 2 & 0,8 \\
\hline & Ensino Secundário & 52 & 21,1 \\
\hline & Licenciatura & 78 & 31,6 \\
\hline & Mestrado & 88 & 35,6 \\
\hline & Doutoramento & 21 & 8,5 \\
\hline \multirow{6}{*}{ Estado civil } & Solteira(o) & 95 & 38,5 \\
\hline & União de facto & 35 & 14,2 \\
\hline & Casada(o) & 95 & 38,5 \\
\hline & Viúva(o) & 1 & 0,4 \\
\hline & Separada(o) & 2 & 0,8 \\
\hline & Divorciada(o) & 19 & 7,7 \\
\hline
\end{tabular}




\section{Instrumentos}

\section{Questionário Sociodemográfico}

O protocolo de recolha de dados incluiu um questionário sociodemográfico, construído pelos autores, com o objetivo de caracterização da amostra, que englobava questões como sexo, idade e habilitações literárias.

\section{Escala de Ansiedade, Depressão e Stress - 21 itens (EADS-21)}

A EADS-21 foi construída por Lovibond e Lovidond em 1995 (tal como citado por Pais-Ribeiro et al., 2004), e traduzida para a população portuguesa por Pais-Ribeiro et al. (2004). Tem como objetivo a avaliação da presença de afeto negativo a partir de três subescalas: ansiedade, depressão e stress, constituídas por sete itens cada uma, perfazendo assim o total dos 21 itens. A primeira subescala avalia a depressão, englobando sintomas de disforia, desânimo, desvalorização, anedonia e inércia. A segunda subescala centra-se na excitação do sistema nervoso autónomo, ansiedade situacional e experiências ansiógenas subjetivas, dedicando-se por isso, à avaliação da ansiedade. Por último, a subescala do stress foca-se na dificuldade em relaxar, na impaciência e na irritabilidade face a reações exageradas. As opções de resposta distribuem-se numa escala tipo Likert e variam entre zero (não se aplicou nada a mim) e três (aplicou-se a mim a maior parte das vezes), representando assim a severidade e frequência dos sintomas experienciados nos últimos sete dias. A pontuação resulta num somatório dos pontos inerentes a cada opção de resposta, oscilando assim entre os zero e os 21 pontos para cada uma das três subescalas, sendo que estados mais negativos estão associados a pontuações mais elevadas.

\section{Mental Health Continuum - Short Form (MHC-SF)}

De forma a avaliar as dimensões do bem-estar utilizou-se o MHC-SF, desenvolvido por Keyes (2005, tal como citado por Fonte et al. 2020) e adaptado para a população portuguesa por Fonte et al. (2020). Trata-se de um questionário de autorresposta, constituído por 14 itens, distribuídos em três escalas: bem-estar emocional ( 3 itens), bem-estar psicológico (6 itens) e bem-estar social (5 itens). As opções de resposta variam de zero (nunca) a cinco (todos os dias), numa escala tipo Likert, sendo que pontuações mais elevadas são indicadoras de maior bem-estar.

\section{Inventário de Psicoterapia Positiva (IPP)}

Por último, procedeu-se à administração do IPP, desenvolvido por Rashid e Anjum (2008), nos EUA, tendo por base a teoria do bem-estar e as três dimensões que a compõe: vida significativa, vida agradável e vida comprometida. Demonstra-se uma mais-valia para a prática clínica, na medida em que permite aos profissionais de saúde mental avaliar de que forma o processo terapêutico contribuiu para alteração das perceções do sujeito face às dimensões do bem-estar, descurando assim o foco do que é estritamente patológico. Trata-se de um inventário de autorresposta, composto por 25 itens, organizados de cinco (muito parecido comigo) até um (eu não sou nada assim), numa escala tipo Likert invertida. A cotação é realizada através de somatório, sendo que pontuações mais elevadas são indicadoras de bem-estar e felicidade plena. O IPP encontra-se validado em países como a Turquia (Guney, 2011), Alemanha (Khanjani et al., 2014) e Irão (Wammerl et al., 2015), apresentando boas qualidades psicométricas testadas do ponto de vista da validade e fidelidade da escala. 


\section{Procedimento}

Com vista à sustentação teórica da validação do instrumento foi realizada uma revisão da literatura sobre bemestar e psicoterapia positiva, garantindo que o IPP não se encontrava já validado para a população portuguesa. Posteriormente foi estabelecido o contacto com os autores e solicitada autorização para a validação, que concederam parecer favorável.

Após submissão e aprovação do projeto pela comissão de ética da Universidade Fernando Pessoa, onde o projeto se desenvolveu, e já obtidas as autorizações necessárias para a utilização dos instrumentos, avançou-se numa primeira fase para a tradução da versão original do IPP em inglês. Este foi traduzido de modo independente por dois portugueses nativos fluentes em inglês, com vista à obtenção de uma única versão após discussão. A versão resultante desta análise foi retrovertida para inglês por um inglês nativo fluente em Português e verificada equivalência semântica dos itens.

Em seguida, procedeu-se a uma análise e reflexão falada dos itens do questionário com um pequeno grupo de participantes de diferentes idades e escolaridade, a fim de analisar a clareza e a compreensão dos mesmos (ca. 20 participantes, jovens adultos, adultos e idosos).

A estes participantes foi solicitado o consentimento informado e foram indivíduos da rede de contactos das investigadoras, com administração presencial.

Posteriormente, foi desenvolvido o questionário online, com recurso à plataforma de construção de questionários online Google Docs.

Todas as informações acerca do estudo foram contempladas, assim como a confidencialidade e anonimato dos dados e a ausência de quaisquer despesas ou consequências em caso de desistência do participante. A voluntariedade da participação foi garantida, através de um botão de aceitação que teria de ser acionado pelos participantes, sem o qual o questionário não ficaria acessível.

Foi ainda assegurado que os dados recolhidos apenas seriam acedidos pelos investigadores e destruídos no final do estudo.

A recolha de dados decorreu entre Junho e Setembro de 2019, através da divulgação online do link do estudo criado previamente.

\section{Análise Estatística}

A análise e o tratamento de dados foram realizados recorrendo ao programa Statistical Package for the Social Sciences (SPSS), versão 25. O campo omisso foi preenchido com o valor de 999 , de forma a garantir que não existissem coincidências com um valor válido previamente inserido, interferindo com a análise dos dados (Martins, 2011).

Recorreu-se à estatística descritiva (médias, desvio-padrão, moda, mínimo e máximo) para a caracterização da amostra e identificação de valores válidos e omissos face ao IPP e restantes escalas administradas (Espírito-Santo \& Daniel, 2017).

Quanto às propriedades psicométricas, ao nível da confiabilidade, procedeu-se à análise do alfa de Cronbach, splithalf e correlações item com o total do teste (Daniel et al., 2015). No que toca à validade da escala, recorreu-se à realização da análise fatorial e validade convergente e divergente (Ribeiro, 2010).

Para todos os procedimentos estatísticos adotados o valor de alfa estabelecido foi de 0,05 - o mais utilizado em estudos exploratórios (Espírito-Santo \& Daniel, 2015). 


\section{Resultados}

\section{Análises descritivas}

Atendendo aos resultados das análises descritivas, tendo em conta os valores mínimos e máximos passíveis de atingir, pode verificar-se que a média do IPP é de 96,62 ( $D P=21,29)$. As pontuações médias obtidas para as subescalas da EADS-21 e do MHC-SF sugerem baixos níveis de ansiedade, depressão e stress moderado, bem-estar psicológico, social e emocional, respetivamente. Não existem valores omissos, pelo que, os resultados remetem para a amostra total constituída por 247 sujeitos.

\section{Propriedades Psicométricas}

\section{Consistência Interna}

A versão portuguesa do IPP apresentou um alfa de Cronbach de 0,97, evidenciando assim o nível elevado de consistência interna (Daniel et al., 2015).

\section{Teste das Duas Metades ou Bipartição (Split-Half)}

Observando a Tabela 2 compreende-se que o IPP apresentou altos valores de correlação entre formas $(r=0,92)$, mantendo o nível elevado de consistência interna em cada uma das metades ( $\alpha$ de Cronbach $=0,95$; $\alpha$ de Cronbach $=0,92)$. Tais resultados foram obtidos através do teste split-half que se propõe medir a correlação entre as pontuações das duas metades do instrumento (Espírito-Santo, 2017; Espirito-Santo \& Daniel, 2017).

\section{Tabela 2}

Correlações Entre as Duas Metades do IPP numa Amostra da População Portuguesa (N = 247)

\begin{tabular}{|c|c|c|c|}
\hline \multirow{5}{*}{ Alfa de Cronbach } & \multirow{2}{*}{ Parte 1} & Valor & 0,95 \\
\hline & & $N$ de itens & $13^{\mathrm{a}}$ \\
\hline & \multirow{2}{*}{ Parte 2} & Valor & 0,93 \\
\hline & & $N$ de itens & $12^{\mathrm{b}}$ \\
\hline & \multicolumn{2}{|c|}{$N$ total de itens } & 25 \\
\hline \multicolumn{3}{|l|}{ Correlação entre formas } & 0,92 \\
\hline \multirow{2}{*}{ Coeficiente de Spearman-Brown } & \multicolumn{2}{|c|}{ Comprimento igual } & 0,96 \\
\hline & \multicolumn{2}{|c|}{ Comprimento desigual } & 0,96 \\
\hline \multicolumn{3}{|c|}{ Coeficiente das duas metades de Gutman } & 0,96 \\
\hline
\end{tabular}

Nota. IPP = Inventário de Psicoterapia Positiva. ${ }^{a}$ Os 13 primeiros itens do IPP. ${ }^{\mathrm{b}}$ Os restantes 12 itens.

\section{Correlação do Item com o Total da Escala}

Por último, foi realizada a matriz de correlação entre os itens da escala - correlação do item com o total do teste - que permite compreender o padrão de relacionamento entre as variáveis através do valor de $r$ de Pearson (Espinoza-Venegas et al., 2015).

Os resultados evidenciados na Tabela 3 demonstram que as correlações dos itens com o total do IPP oscilam entre 0,57 e 0,89, apresentando, deste modo, valores de correlação consideráveis/altos (Espirito-Santo \& Daniel, 2017). 


\section{Tabela 3}

Correlação do Item com o Total do IPP e Análise Fatorial Exploratória com Rotação Varimax e Comunalidades numa Amostra da População Portuguesa ( $=247)$

\begin{tabular}{|c|c|c|c|c|c|}
\hline \multirow[b]{2}{*}{ Itens IPP } & \multirow{2}{*}{$\begin{array}{c}\text { Total IPP } \\
r \\
\end{array}$} & \multicolumn{3}{|c|}{ Componentes } & \multirow{2}{*}{$h^{2}$} \\
\hline & & 1 & 2 & 3 & \\
\hline Item 1 & $0,81^{* * *}$ & & 0,75 & & 0,8 \\
\hline Item 2 & $0,83^{* * *}$ & & 0,45 & & 0,7 \\
\hline Item 3 & $0,78^{* * *}$ & 0,64 & & & 0,6 \\
\hline Item 4 & $0,70^{* * *}$ & & & 0,40 & 0,6 \\
\hline Item 5 & $0,61^{* * *}$ & 0,60 & & & 0,4 \\
\hline Item 6 & $0,80^{* * *}$ & & 0,56 & & 0,7 \\
\hline Item 7 & $0,82^{* * *}$ & 0,72 & & & 0,7 \\
\hline Item 8 & $0,76^{* * *}$ & 0,76 & & & 0,6 \\
\hline Item 9 & $0,85^{* * *}$ & 0,71 & & & 0,7 \\
\hline Item 10 & $0,75^{* * *}$ & 0,68 & & & 0,6 \\
\hline Item 11 & $0,85^{* * *}$ & 0,73 & & & 0,7 \\
\hline Item 12 & $0,88^{* * *}$ & & 0,41 & & 0,8 \\
\hline Item 13 & $0,80^{* * *}$ & 0,80 & & & 0,7 \\
\hline Item 14 & $0,83^{* * *}$ & & & 0,87 & 0,8 \\
\hline Item 15 & $0,80^{* * *}$ & 0,75 & & & 0,7 \\
\hline Item 16 & $0,57^{* * *}$ & & 0,86 & & 0,8 \\
\hline Item 17 & $0,83^{* * *}$ & & 0,47 & & 0,7 \\
\hline Item 18 & $0,87^{* * *}$ & 0,80 & & & 0,8 \\
\hline Item 19 & $0,76^{* * *}$ & 0,67 & & & 0,7 \\
\hline Item 20 & $0,83^{* * *}$ & 0,80 & & & 0,7 \\
\hline Item 21 & $0,79^{* * *}$ & & 0,64 & & 0,7 \\
\hline Item 22 & $0,87^{* * *}$ & 0,80 & & & 0,8 \\
\hline Item 23 & $0,85^{* * *}$ & 0,82 & & & 0,8 \\
\hline Item 24 & $0,85^{* * *}$ & 0,86 & & & 0,8 \\
\hline Item 25 & $0,89^{* * *}$ & 0,88 & & & 0,8 \\
\hline
\end{tabular}

Nota. IPP = Inventário de Psicoterapia Positiva; $r=$ correlação; $h^{2}=$ comunalidade.

$* * * p<0,001$.

\section{Análise Fatorial}

Para a extração de fatores recorreu-se à análise fatorial exploratória com rotação ortogonal Varimax, respeitando os procedimentos adotados pelos autores nos restantes países para os quais o IPP se encontra validado (Guney, 2011; Khanjani et al., 2014; Wammerl et al., 2015), O teste de Kaiser-Meyer-Olkin (KMO) de adequação da amostra foi de 0,96 e o teste de esfericidade de Bartlett foi significativo $(p<0,001)$, cumprindo assim os 
pressupostos para a realização da análise fatorial. Relativamente às comunalidades, os valores obtidos oscilaram entre os 0,3 e os 0,8 evitando a supressão de qualquer dos 25 itens que compõem o IPP.

Foram extraídos três fatores que explicaram $70,6 \%$ da escala. 0 Fator 1 apresentou uma variância explicada de $48,0 \%$ e correspondeu à dimensão da vida significativa, sendo composto por 16 itens $(3,5,7,8,9,10,11,13,15$, 18, 19, 20, 22, 23, 24 e 25). O Fator 2 explicou 17,7\% da escala, agrupou sete itens $(1,2,6,12,16$, 17 e 21) e correspondeu à dimensão vida agradável. Por último, o Fator 3 apresentou uma variância explicada de 4,9\%, denominando-se vida comprometida e agrupou dois itens (4 e 14). A Tabela 3 resume os resultados obtidos e descritos na análise fatorial.

\section{Validade Convergente e Divergente}

Como se pode observar na Tabela 4, o IPP apresentou uma correlação positiva significativa moderada com as subescalas do MHC-SF. Por outro lado, as correlações discriminaram de forma negativa significativa baixa com as subescalas da EADS-21 (Espirito-Santo \& Daniel, 2017).

\section{Tabela 4}

Correlações Entre o IPP e a MHC-SF e EADS-21 para Validade Convergente e Divergente $(\mathrm{N}=247)$

\begin{tabular}{llll}
\hline Validade convergente & $r$ & Validade divergente & $r$ \\
\hline MHC-SF (BE) & $0,32^{*}$ & EADS-21 (Ansiedade) & $-0,26^{*}$ \\
MHC-SF (BS) & $0,23^{*}$ & EADS-21 (Depressão) & $-0,10^{*}$ \\
MHC-SF (BP) & $0,32^{*}$ & EADS-21 (Stress) & $-0,19^{*}$ \\
\hline
\end{tabular}

Nota. IPP = Inventário de Psicoterapia Positiva; MHC-SF = Mental Health Continuum - Short Form; BE = bem-estar emocional; BS = bem-estar social; $\mathrm{BP}=$ bem-estar psicológico; EADS-21 = Escala de Ansiedade, Depressão e Stress - 21 itens.

$* p<0,05$.

\section{Discussão}

O presente artigo teve por objetivo apresentar uma versão preliminar dos resultados da tradução e validação do IPP para a população portuguesa. Os procedimentos adotados para a versão portuguesa do IPP seguiram as orientações dos restantes países para os quais já se encontra aferido.

A amostra total foi constituída por 247 participantes, maioritariamente do sexo feminino, com idades entre os 18 e os 69 anos. O mestrado é o nível académico mais proeminente, registando-se uma percentagem igualitária entre solteiros e casados no que concerne ao estado civil. Compreende-se, deste modo, que o número total de participantes é inferior ao dos restantes países para os quais o IPP já se encontra validado. No entanto, trata-se de um estudo preliminar pelo que, a amostra será ainda alargada. Uma vez que a recolha de dados foi realizada por via online, as respostas ao IPP foram tornadas obrigatórias, garantindo que não existiam dados omissos. Tal não aconteceu na versão turca que, embora seja constituída por uma amostra alargada de participantes, contém cerca de $5 \%$ de respostas omissas, constituindo assim um entrave para as análises e procedimentos estatísticos. 
De acordo com os resultados obtidos para a mesma população, atendendo aos restantes dados da caracterização sociodemográfica, constata-se que existe um maior predomínio do sexo feminino, tratando-se de uma amostra escolarizada numa faixa etária intermédia (Guney, 2011).

As pontuações médias obtidas através do IPP sugerem que os participantes apresentam níveis moderados de bemestar/felicidade plena corroborados pelas pontuações médias obtidas nas subescalas de bem-estar psicológico, social e emocional do MHC-SF. Concomitantemente, foram obtidos baixos níveis de ansiedade, depressão e stress, medidos através dos resultados médios da EADS-21.

As qualidades psicométricas do IPP foram testadas ao nível da confiabilidade (alfa de Cronbach, split-half e correlação do item com o total da escala) e validade (análise fatorial e validade convergente e divergente).

Relativamente à confiabilidade, o IPP apresentou um nível elevado de consistência interna (Daniel et al., 2015), superior aos valores obtidos na Turquia, Alemanha e Irão (Guney, 2011; Khanjani et al., 2014; Wammerl et al., 2015).

Os resultados obtidos no teste split-half demonstram que o IPP apresenta valores de correlação entre formas superiores aos obtidos na população turca (Guney, 2011).

No que concerne à matriz de correlação entre os itens e o total do teste - procedimento realizado apenas para a versão portuguesa - o IPP apresentou níveis de correlação significativos, consideráveis/altos (Espirito-Santo \& Daniel, 2017). Depreende-se deste modo que, uma vez que existe correlação significativa, os itens medem o mesmo constructo que o total da escala se propõe medir, constatando assim a sua unidimensionalidade (EspinozaVenegas et al., 2015; Ribeiro, 2010).

Relativamente às qualidades psicométricas ao nível da validade, à vista das restantes validações do IPP, foi realizada a análise fatorial exploratória com rotação ortogonal Varimax. Os valores obtidos no teste de KMO e Bartlett permitiu concluir que é possível realizar o procedimento estatístico da análise fatorial no IPP para a amostra que compõe o presente estudo e que todos os itens estão em sintonia com o principal construto medido pela escala (Primi (2012). Os valores obtidos oscilam entre os 0,3 e os 0,8 evitando a supressão de qualquer dos 25 itens que compõem o IPP. Tais pressupostos não foram cumpridos na versão turca uma vez que, a versão final do inventário era constituída por 21 itens, indicando assim a supressão de quatro itens (Guney, 2011). A versão preliminar do IPP para a população portuguesa permitiu extrair três fatores. Atendendo aos construtos teóricos subjacentes ao IPP, compreende-se que o Fator 1 corresponde à dimensão da vida significativa, o Fator 2 diz respeito à vida agradável e o Fator 3 à vida comprometida. Os resultados obtidos vão de encontro às análises estatísticas da versão persa do IPP, uma vez que também foram extraídos três fatores que atendem às dimensões da felicidade plena. Por outro lado, diferenciam-se da população turca, uma vez que foram extraídos cinco fatores nesta versão do IPP, que se enquadram no modelo PERMA (Guney, 2011; Khanjani et al., 2014).

A versão preliminar do IPP para a população portuguesa apresenta uma correlação positiva significativa moderada com as subescalas do MHC-SF (bem-estar emocional, bem-estar social e bem-estar psicológico). Depreende-se deste modo que, quanto maiores os níveis de felicidade plena, maiores serão os níveis de bem-estar emocional, social e psicológico. No que toca à validade divergente, optou-se por correlacionar o IPP com a EADS-21, obtendose correlações negativas significativas baixas com as subescalas da ansiedade, depressão e stress. Assim, quanto maiores os níveis de felicidade plena, menores os níveis de ansiedade, depressão e stress, ou seja, menor predomínio do afeto negativo (Espirito-Santo \& Daniel, 2017). A versão preliminar do IPP cumpre assim os pressupostos da validade convergente e divergente, que não foram calculados para os restantes países para os quais o IPP se encontra aferido. 


\section{Conclusão}

A versão preliminar do IPP para a população portuguesa, apresentou boas qualidades psicométricas testadas ao nível da confiabilidade e validade da escala, superiores às obtidas na Turquia, Alemanha e Irão. No entanto, apesar dos resultados evidenciados ao longo do presente artigo, ressalva-se que se trata de um estudo preliminar, pelo que nos encontramos em fase de recolha e tratamento de dados para uma amostra portuguesa mais alargada. Seguindo as orientações dos estudos realizados nos restantes países sugere-se que o IPP seja validado para populações clínicas específicas uma vez que se demonstra uma mais-valia na avaliação e compreensão dos avanços terapêuticos e eficácia da intervenção psicológica. Estaria assim garantida uma visão holística dos pontos fortes da intervenção psicológica, quer para o utente, quer para o profissional, permitindo uma desconstrução daquele que é o foco no estritamente patológico.

Conflito de interesses | Conflict of interest: nenhum | none.

Fontes de financiamento | Funding sources: nenhuma | none.

Contributos: MF: Contributo na concetualização, definição da metodologia, recolha de dados, tratamento de dados e redação do manuscrito. APe: Contributo na concetualização, definição da metodologia, recolha de dados e tratamento de dados. APr: Contributo na concetualização, definição da metodologia e recolha de dados. CF: Contributo na concetualização, tratamento de dados e redação, revisão e edição da redação final do manuscrito.

\section{Referências}

Asebedo, S., \& Seay, M. C. (2014). Positive psychological attributes and retirement satisfaction. Journal of Financial Counseling and Planning, 25(2), 161-173. https://doi.org/10.2469/dig.v45.n6.1

Daniel, F., Gomes da Silva, A., \& Ferreira, P. (2015). Contributo para a discussão da avaliação da fiabilidade de um instrumento de medição. Revista de Enfermagem Referência, 4(7), 129-137. https://doi.org/10.12707/RIV15003

Espinoza-Venegas, M., Sanhueza-Alvarado, O., Ramírez-Elizondo, N., \& Sáez-Carrillo, K. (2015). Validação do construto e da confiabilidade de uma escala de inteligência emocional aplicada a estudantes de enfermagem. Revista LatinoAmericana de Enfermagem, 23(1), 139-147. https://doi.org/10.1590/0104-1169.3498.2535

Espirito-Santo, H., \& Daniel, F. (2015). Calcular e apresentar tamanhos dos efeitos em trabalhos científicos: As limitações do $\mathrm{p}<0.05$ na análise de diferenças de médias de dois grupos. Revista Portuguesa de Investigação Comportamental e Social, 1(1), 3-16. https://doi.org/10.7342/ismt.rpics.2015.1.1.14

Espirito-Santo, G. (2017). A validade dos instrumentos de avaliação. Revista Portuguesa de Investigação Comportamental e Social, 3(1), 1-1. https://doi.org/10.7342/ismt.rpics.2017.3.1.49

Espirito-Santo, G., \& Daniel, F. (2017). Calcular e apresentar tamanhos dos efeitos em trabalhos científicos: guia para reportar a força das relações. Revista Portuguesa de Investigação Comportamental e Social, 3(1), 53-64. https://doi.org/10.7342/ismt.rpics.2017.3.1.48

Fonte, C., Silva, I., Vilhena, E., \& Keyes, C. L. M. (2020). The Portuguese adaptation of the mental health continuumshort form adult population. Community Mental Health Journal, 56(2), 368-375. https://doi.org/10.1007/s10597019-00484-8

Guney, S. (2011). The positive psychotherapy inventory (PPTI): Reliability and validity study in Turkish population. Procedia - Social and Behavioral Sciences, 29, 81-86. https://doi.org/10.1016/j.sbspro.2011.11.209

Khanjani, M., Shahidi, Sh., FathAbadi, J., Mazaheri, M. A., \& Shokri, O. (2014). The factor structure and psychometric properties of the Positive Psychotherapy Inventory (PPTI) in an Iranian sample. Iranian Journal of Applied Psychology, 7(5), 26-47. https://www.magiran.com/paper/1580927/?lang=en

Kun, A., Balogh, P., \& Krasz, K. (2017). Development of the work-related well-being questionnaire based on seligman's PERMA model. Periodicapolytechnica Social and Management Sciences, 25(1), 56-63. https://doi.org/10.3311/PPso.9326

Martins, C. (2011). Manual de análise de dados quantitativos com recurso ao IBM SPSS. Psiquilíbrios Edições. 
Pais-Ribeiro, J. L., Honrado, A., \& Leal, I. (2004). Contribuição para o estudo da adaptação portuguesa das escalas de ansiedade, depressão e stress (EADS) de 21 itens de Lovibond e Lovibond. Psicologia, Saúde \& Doenças, 5(2), 229239. https://bit.ly/3jzkBli

Primi, R. (2012). Psicometria: Fundamentos matemáticos da teoria clássica dos testes. Avaliação Psicológica, 11(2), 297307. https://bit.ly/34xQZGY

Rashid, T. (2015). Positive psychotherapy: A strength-based approach. The Journal of Positive Psychology, 10(1), 25-40. https://doi.org/10.1080/17439760.2014.920411

Rashid, T., \& Anjum, A. (2008). Positive psychotherapy for young adults and children. Em J. R. Z. Abela \& B. L. Hankin (Eds.), Handbook of depression in children and adolescents (pp. 250-287). Guilford Press.

Rashid, T., \& Seligman, M. (2018). Positive psychotherapy: Workbook. Oxford University Press. https://doi.org/10.1093/med-psych/9780190920241.001.0001

Rashid, T., \& Seligman, M. (2019). Psicoterapia positiva: Artmed Editora.

Ribeiro, J. (2010). Investigação e avaliação em psicologia e saúde (2a ed.). Placebo Editora.

Seligman, M. E. P., Rashid, T., \& Parks, A. C. (2006). Positive psychotherapy. American Psychologist, 61(8), 774-788. https://doi.org/10.1037/0003-066X.61.8.774

Sirgy, M. J., \& Wu, J. (2009). The pleasant life, the engaged life, and the meaningful life: What about the balanced life? Journal of Happiness Studies, 10, 183-196. https://doi.org/10.1007/s10902-007-9074-1

Wammerl, M., Jaunig, J., Maierunteregger, T., \& Streit, P. (2015). The development of German version of the Positive Psychotherapy Inventory Überschrift (PPTI) and the PERMA-Profiler [Paper presentation]. World Congress of International Positive Psychology Association, Orlando, FL, United States. 